\title{
Cardiovascular Pathology
}

National Cancer Institute

\section{Source}

National Cancer Institute. Cardiovascular Pathology. NCI Thesaurus. Code C18273.

Cardiovascular Pathology; a subdiscipline of pathology focusing on diseases of the heart or circulatory system. 